\title{
BARThez: a Skilled Pretrained French Sequence-to-Sequence Model
}

\author{
Moussa Kamal Eddine \\ École Polytechnique
}

\author{
Antoine J.-P. Tixier \\ École Polytechnique
}

\author{
Michalis Vazirgiannis \\ École Polytechnique
}

\begin{abstract}
Inductive transfer learning has taken the entire NLP field by storm, with models such as BERT and BART setting new state of the art on countless NLU tasks. However, most of the available models and research have been conducted for English. In this work, we introduce BARThez, the first large-scale pretrained seq2seq model for French. Being based on BART, BARThez is particularly well-suited for generative tasks. We evaluate BARThez on five discriminative tasks from the FLUE benchmark and two generative tasks from a novel summarization dataset, OrangeSum, that we created for this research. We show BARThez to be very competitive with state-of-the-art BERT-based French language models such as CamemBERT and FlauBERT. We also continue the pretraining of a multilingual BART on BARThez' corpus, and show our resulting model, mBARThez, to significantly boost BARThez' generative performance. Code, data and models are publicly available.
\end{abstract}

\section{Introduction}

Inductive transfer learning, that is, solving tasks with models that have been pretrained on very large amounts of data, was a game changer in computer vision (Krizhevsky et al., 2012). In NLP, while annotated data are scarce, raw text is virtually unlimited and readily available. It thus emerged that the ability to learn good representations from plain text could greatly improve general natural language understanding.

Trained on gigantic amounts of raw data and with hundreds of GPUs, models based on the Transformer architecture (Vaswani et al., 2017), such as GPT (rad) and BERT (Devlin et al., 2019), have set new state-of-the-art performance in every NLU task. Moreover, users around the world can easily benefit from these improvements, by finetuning the publicly available pretrained models to their specific applications. This also saves considerable amounts of time, resources and energy, compared with training models from scratch.

BART (Lewis et al., 2020) combined a BERTliked bidirectional encoder with a GPT-like forward decoder, and pretrained this seq2seq architecture as a denoising autoencoder with a more general formulation of the masked language modeling objectives of BERT. Since not only BART's encoder but also its decoder is pretrained, BART excels on tasks involving text generation.

While the aforementioned efforts have made great strides, most of the research and resources were dedicated to the English language, despite a few notable exceptions. In this paper, we partly address this limitation by contributing BARThez ${ }^{1}$, the first pretrained seq2seq model for French.

BARThez, based on BART, was pretrained on a very large monolingual French corpus from past research that we adapted to suit BART's specific perturbation schemes. Unlike already existing BERTbased French language models such as CamemBERT (Martin et al., 2020) and FlauBERT (Le et al., 2020), BARThez is particularly well-suited for generative tasks. We evaluate BARThez on five sentiment analysis, paraphrase identification, and natural language inference tasks from the recent FLUE benchmark, and two generative tasks from a novel French summarization dataset, OrangeSum, that we created for this research. We also continue the pretraining of an already pretrained multilingual BART on BARThez' corpus. Our resulting model, mBARThez, significantly boosts BARThez' performance on generative tasks.

Our contributions are as follows:

- We publicly release the first large-scale pretrained seq2seq model dedicated to the French language, BARThez, featuring $165 \mathrm{M}$ parameters, and trained on $101 \mathrm{~GB}$ of text for 60 hours with 128 GPUs.

\footnotetext{
${ }^{1}$ named after a legendary French goalkeeper, Fabien Barthez: https://en.wikipedia.org/wiki/Fabien_Barthez
} 
We evaluate BARThez on five discriminative tasks and two generative tasks, with automated and human evaluation, and show that BARThez is very competitive with the state of the art.

- To address the lack of generative tasks in the existing FLUE benchmark, we put together a novel dataset for summarization in French, OrangeSum, that we publicly release ${ }^{2}$ and analyze in this paper. OrangeSum is more abstractive than traditional summarization datasets, and can be considered the French equivalent of XSum (Narayan et al., 2018). - We continue the pretraining of a multilingual BART on BARThez' corpus, and show that our resulting model, named mBARThez, offers a significant boost over BARThez on generative tasks.

- We publicly release our code and models ${ }^{3}$. Our models were also integrated into the highly-popular Hugging Face Transformers library ${ }^{4}$. As such, they can easily be distributed and deployed for research or production within a standard, industrialstrength framework. They also have their own APIs and can be interactively tested online.

\section{Related work}

Learning without labels is enabled via selfsupervised learning ${ }^{5}$, a setting in which a system learns to predict part of its input from other parts of its input. In practice, one or more supervised tasks are created from the unlabeled data, and the model learns to solve these tasks with custom objectives.

Some of the earliest and most famous selfsupervised representation learning approaches in NLP are word2vec (Mikolov et al., 2013), GloVe (Pennington et al., 2014) and FastText (Bojanowski et al., 2017). While these methods were significant advancements, they produce static representations, which is a major limitation, as words have different meanings depending on the unique contexts in which they are used.

Deep pretrained language models. ELMo (Peters et al., 2018) provided the first contextualized embeddings, by extracting and combining the internal states of a pretrained deep bi-LSTM language model. Except for the word embeddings and the softmax layer, the forwards and backwards RNNs have different parameters. The authors of ELMo showed that the learned representations could be

\footnotetext{
${ }^{2}$ https://github.com/Tixierae/OrangeSum

${ }^{3}$ https://github.com/moussaKam/BARThez

${ }^{4}$ https://huggingface.co/moussaKam

${ }^{5}$ a term coined by Yann LeCun.
}

transferred with great benefits to downstream architectures, to solve a variety of supervised NLU tasks.

Beyond simply combining internal states, Howard and Ruder (2018) proposed ULMFiT, a universal transfer learning method for text classification where the language model is pretrained on a large, general dataset, finetuned on a specific dataset, and finally augmented with classification layers trained from scratch on downstream tasks.

With the OpenAI GPT, rad capitalized on the Transformer architecture (Vaswani et al., 2017), superior and conceptually simpler than recurrent neural networks. More precisely, they pretrained a left-to-right Transformer decoder as a general language model, and finetuned it on 12 language understanding tasks by applying different transformations to the input.

By combining ideas from all the aforementioned models, and introducing bidirectional pretraining, BERT (Devlin et al., 2019) disrupted the NLP field by setting new state-of-the-art performance on 11 NLU tasks, with very wide margins. More precisely, BERT uses a bidirectional Transformer encoder with a masked language model objective, making the learned representations capture both the left and the right contexts, instead of just the left context. The sheer size of BERT, with up to 24 Transformer blocks, plays a role in performance too.

With GPT-2, a version of GPT with over an order of magnitude more parameters than GPT, Radford et al. (2019) showed that as long as they have very large capacities, general language models can reach reasonable performance on many specific NLU tasks out-of-the-box, without any finetuning, i.e., accomplish zero-shot transfer. This demonstrates the fundamental nature and importance of the language modeling objective for inductive transfer learning.

In RoBERTa, Liu et al. (2019) showed that the performance of BERT could be improved by optimizing its hyperparameters and training procedure. The study of why and how BERT works so well has now its own dedicated research field, known as BERTology (Rogers et al., 2020).

Languages. Following the success of BERT for the English language, some BERT models were pretrained and evaluated in other languages. Some examples include Arabic (Antoun et al., 2020), Dutch (de Vries et al., 2019; Delobelle et al., 2020), 
French (Martin et al., 2020; Le et al., 2020), Italian (Polignano et al., 2019), Portuguese (Souza et al., 2019), Russian (Kuratov and Arkhipov, 2019), and Spanish (Cañete et al., 2020).

In addition to the aforelisted monolingual models, multilingual models were also proposed, notably mBERT (Devlin et al., 2019), XLM (Conneau and Lample, 2019) and XLM-R (Conneau et al., 2020).

Abstractive summarization. Abstractive summarization is an important and challenging task, requiring diverse and complex natural language understanding and generation capabilities. A good summarization model needs to read, comprehend, and write well.

GPT-2 can be used for summarization, by sampling a certain numbers of tokens from a given start seed. However, while the generated text is grammatical and fluent, summarization performance is only slightly superior to that of a random extractive baseline.

Being a bidirectional encoder, BERT cannot be used out-of-the-box for language generation, unlike GPT-2. Furthermore, BERT produces singlesentence representations, whereas for summarization, reasoning over multiple sentence and paragraph representations is necessary. Liu and Lapata (2019) proposed a way to overcome these challenges. At the input level, they introduced special tokens to encode individual sentences, interval segment embeddings, and used more position embeddings than in BERT. Then, they combined a pretrained BERT encoder with a Transformer-based decoder initialized at random and jointly trained the two models with different optimizers and learning rates.

BART and mBART. BART (Lewis et al., 2020) is a denoising auto-encoder that jointly pretrains a bidirectional encoder (like in BERT) and a forward decoder (like in GPT) by learning to reconstruct a corrupted input sequence. Both the encoder and the decoder are Transformers. Since not only the encoder but also the decoder is pretrained, BART is particularly effective when applied to text generation tasks.

Liu et al. (2020) pretrained a multilingual BART (mBART) on 25 different languages. They showed that this multilingual pretraining brings significant performance gains on a variety of machine translation tasks. MASS (Song et al., 2019) is another multilingual pretrained sequence to sequence model, that learns to predict a masked span in the input sequence. The main difference between MASS and BART, is that the former only predicts the masked fragment of the sentence, while the latter learns to reconstruct the entire corrupted sentence. This difference makes MASS less effective in discriminative tasks, given that only the masked span is fed to the decoder (Lewis et al., 2020). ProphetNet (Qi et al., 2020) which also adopts the encoderdecoder structure, introduces a new learning objective called future $\mathrm{n}$-gram prediction. This objective reduces overfitting on local correlations by learning to predict the next $n$-grams (instead of unigrams) at each time step given the previous context

\section{BARThez}

Our model is based on BART (Lewis et al., 2020), a denoising auto-encoder. It consists of a bidirectional encoder and a left-to-right auto-regressive decoder.

\subsection{Architecture}

We use the BASE architecture, with 6 encoder and 6 decoder layers. We did not opt for a LARGE architecture due to resource limitations. Our BASE architecture uses 768 hidden dimensions and 12 attention heads in both the encoder and the decoder. In total, our model has roughly $165 \mathrm{M}$ parameters. The architecture has two differences compared with the vanilla seq2seq Transformer (Vaswani et al., 2017). The first one is the use of GeLUs activation layers instead of ReLUs, and the second is the presence of a normalization layer on top of the encoder and the decoder, following Liu et al. (2020). These additional layers help stabilizing the training when using FP16 precision.

\subsection{Vocabulary}

To generate our vocabulary, we use SentencePiece (Kudo and Richardson, 2018) that implements bytepair-encoding (BPE) (Sennrich et al., 2016). We do not perform any type of pre-tokenization and we fix the size of the vocabulary to $50 \mathrm{~K}$ sub-words. The SentencePiece model is trained on a 10GB random sample of the pretraining corpus. We fix the character coverage to $99.95 \%$.

\subsection{Self-supervised learning}

We use the same pretraining as in BART. That is, BARThez learns to reconstruct an input sequence that was perturbed with a noise function. BARThez' 
objective is to minimize the cross-entropy between the predicted and the original text. Formally, having a set of documents $\left\{X_{1}, X_{2}, \ldots, X_{n}\right\}$ and a noising function $n$, we aim at finding the parameters $\theta$ that minimize:

$$
L_{\theta}=-\sum_{i} \log P\left(X_{i} \mid n\left(X_{i}\right) ; \theta\right)
$$

Following Lewis et al. (2020), we apply two types of noise in $n$. First, we use text infilling, where a number of text spans are sampled and replaced with one [MASK] special token. The length of the spans is sampled from a Poisson distribution with $(\lambda=3.5)$ and $30 \%$ of the text is masked. Second, we use sentence permutation, where the input document, seen as a list of sentences, is shuffled.

\subsection{Pretraining corpus}

We created a version of FlauBERT's corpus (Le et al., 2020) suitable for the two perturbation schemes described in subsection 3.3. Indeed, in the original FlauBERT corpus, each sentence is seen as an independent instance, while in our case, we need complete documents as instances.

Other than that, BARThez' corpus is similar to FlauBERT's. It primarily consists in the French part of CommonCrawl, NewsCrawl, Wikipedia and other smaller corpora that are listed in Table 1. To clean the corpus from noisy examples, we used the script $^{6}$ provided by Le et al. (2020). Note that we disabled the Moses tokenizer, as we used SentencePiece, which does not require any pre-tokenization. The total corpus size was $66 / 101$ GB before/after SentencePiece tokenization.

\begin{tabular}{|l|c|}
\hline Corpus & Size \\
\hline CommonCrawl & 42.0 \\
NewsCrawl (Li et al., 2019) & 9.6 \\
Wikipedia & 4.0 \\
GIGA (Tiedemann, 2012) & 3.8 \\
ORTOLANG (ATILF and CLLE, 2020) & 2.7 \\
MultiUn (Eisele and Chen, 2010) & 2.2 \\
EU Bookshop (Skadiňs et al., 2014) & 2.1 \\
\hline
\end{tabular}

Table 1: BARThez' corpora (in GB, after cleaning).

\subsection{Training details}

We pretrained BARThez on 128 NVidia V100 GPUs. We fixed the batch size to 6000 tokens per GPU and the update frequency to 2 , which gave a total number of roughly $22 \mathrm{k}$ documents per update. We used the Adam optimizer (Kingma

\footnotetext{
${ }^{6}$ https://github.com/getalp/Flaubert
}

and $\mathrm{Ba}$, 2015) with $\epsilon=10^{-6}, \beta_{1}=0.9$, and $\beta_{2}=0.999$, with a learning rate starting from $6.10^{-4}$ and decreasing linearly as a function of the training step. We used a warm up of $6 \%$ of the total number of training steps. Pretraining lasted for approximately 60 hours, allowing for 20 passes over the whole corpus. In the first 12 epochs, we fixed the dropout to 0.1 , for epochs 12 to 16 we decreased it to 0.05 , and finally we set it to zero for epochs 16 to 20. All experiments were carried out using the Fairseq library (Ott et al., 2019).

\section{4 mBARThez}

mBART (Liu et al., 2020) is a multilingual BART. It follows a LARGE architecture, with 12 layers in both the encoder and the decoder, hidden vectors of size 1024, and 16 attention heads. It was trained on a multilingual corpus containing 1369 GB of raw text, for over 2.5 weeks on 256 Nvidia V100 GPUs. The multilingual corpus covers 25 different languages, including 56 GB of French text. In the original paper, the authors evaluated mBART on machine translation. However, mBART can also be used to perform monolingual tasks.

We continued the pretraining of the pretrained mBART on BARThez' corpus (see subsection 3.4) for about 30 hours on 128 Nvidia V100 GPUs, which allowed for 4 passes over BARThez' corpus. This can be seen as an instance of languageadaptive pretraining, which goes a step further than domain-adaptive pretraining (Gururangan et al., 2020). The initial learning rate was set to $10^{-4}$ and linearly decreased towards zero. We call the resulting model mBARThez.

Note that we eliminated the tokens containing non-latin characters from mBARThez' embedding layers, reducing its number of parameters from $610 \mathrm{M}$ to $458 \mathrm{M}$.

\section{OrangeSum}

BART-based models are particularly well-suited to generative tasks, but FLUE (Le et al., 2020), the French equivalent of GLUE, only contains discriminative tasks ${ }^{7}$ (Wang et al., 2019b).

We therefore decided to create one such task. We opted for single-document abstractive summarization, as it is a generative task that also requires the model to encode its input very well. In other words, for a model to summarize well, it needs

\footnotetext{
${ }^{7}$ There is no generative task in GLUE or superGLUE (Wang et al., 2019a) either.
} 


\begin{tabular}{|l|c|cc|cc|cc|}
\hline \multirow{2}{*}{ Dataset } & \multirow{2}{*}{ Train/val/test } & \multicolumn{2}{|c|}{ Avg. doc length } & \multicolumn{2}{c|}{$\begin{array}{c}\text { Avg. Summary length } \\
\text { Words }\end{array}$} & Sentences & Wocab size \\
& & & Words & Sentences & Docs & Summaries \\
\hline CNN & $90.3 / 1.22 / 1.09$ & 760.50 & 33.98 & 45.70 & 3.58 & 34 & 89 \\
DailyMail & $197 / 12.15 / 10.40$ & 653.33 & 29.33 & 54.65 & 3.86 & 564 & 180 \\
NY Times & $590 / 32.73 / 32.73$ & 800.04 & 35.55 & 45.54 & 2.44 & 1233 & 293 \\
\hline XSum & $204 / 11.33 / 11.33$ & 431.07 & 19.77 & 23.26 & 1.00 & 399 & 81 \\
OrangeSum Title & $30.6 / 1.5 / 1.5$ & 315.31 & 10.87 & 11.42 & 1.00 & 483 & 43 \\
OrangeSum Abstract & $21.4 / 1.5 / 1.5$ & 350 & 12.06 & 32.12 & 1.43 & 420 & 71 \\
\hline
\end{tabular}

Table 2: Sizes (column 2) are given in thousands of documents. Document and summary lengths are in words. Vocab sizes are in thousands of tokens.

\begin{tabular}{|l|cccc|ccc|ccc|}
\hline \multirow{2}{*}{ Dataset } & \multicolumn{4}{|c}{ \% of novel n-grams in gold summary } & \multicolumn{3}{c|}{ LEAD } & \multicolumn{3}{c|}{ EXT-ORACLE } \\
& Unigrams & Bigrams & Trigrams & 4-grams & R-1 & R-2 & R-L & R-1 & R-2 & R-L \\
\hline CNN & 16.75 & 54.33 & 72.42 & 80.37 & 29.15 & 11.13 & 25.95 & 50.38 & 28.55 & 46.58 \\
DailyMail & 17.03 & 53.78 & 72.14 & 80.28 & 40.68 & 18.36 & 37.25 & 55.12 & 30.55 & 51.24 \\
NY Times & 22.64 & 55.59 & 71.93 & 80.16 & 31.85 & 15.86 & 23.75 & 52.08 & 31.59 & 46.72 \\
\hline XSum & 35.76 & 83.45 & 95.50 & 98.49 & 16.30 & 1.61 & 11.95 & 29.79 & 8.81 & 22.65 \\
OrangeSum Title & 26.54 & 66.70 & 84.18 & 91.12 & 19.84 & 08.11 & 16.13 & 31.62 & 17.06 & 28.26 \\
OrangeSum Abstract & 30.03 & 67.15 & 81.94 & 88.3 & 22.21 & 07.00 & 15.48 & 38.36 & 20.87 & 31.08 \\
\hline
\end{tabular}

Table 3: Degree of abstractivity of OrangeSum compared with that of other datasets, as reported in Narayan et al. (2018). It can be observed that XSum and OrangeSum are more abstractive than traditional summarization datasets.

to both read, comprehend, and write well, making abstractive summarization one of the most central and challenging evaluation tasks in NLP.

Motivation. Our strategy here was to create a French equivalent of the recently introduced XSum dataset (Narayan et al., 2018). XSum was created by scraping articles and their one-sentence summaries from the BBC website, where the summaries are not catchy headlines, but capture the gist of the articles. Unlike the historical CNN, DailyMail, and NY Times datasets (Hermann et al., 2015), which favor extractivity, performing well on XSum requires a high degree of abstractivity.

Data collection. We adopted an analogous strategy, and scraped the "Orange Actu" website ${ }^{8}$. Orange S.A. is a large French telecommunications corporation, with $266 \mathrm{M}$ customers worldwide. Our scraped pages cover almost a decade from Feb 2011 to Sep 2020. They belong to five main categories: France, world, politics, automotive, and society 9 . The society category is divided into 8 subcategories: health, environment, people, culture, media, hightech, unusual ("insolite" in French), and miscellaneous.

Each article featured a single-sentence title as well as a very brief abstract, both professionally written by the author of the article. We extracted these two fields from each page, thus creating two summarization tasks: OrangeSum Title and Or-

\footnotetext{
${ }^{8}$ https: / / actu.orange.fr/, 'Actu' means News.

${ }^{9}$ root URLs are https: / / actu.orange.fr/ for all categories except https://auto.orange.fr/news/ for automotive.
}

angeSum Abstract. Gold summaries are respectively 11.42 and 32.12 words in length on average (see Table 2). Note that like in XSum, titles in OrangeSum tend not to be catchy headlines but rather convey the essence of the article. The same can be said about the abstracts.

Post-processing. As a post-processing step, we removed all empty articles, and articles whose titles were shorter than 5 words. For OrangeSum Abstract, we removed the top $10 \%$ articles in terms of proportion of novel unigrams in the abstracts, as we observed that such abstracts tended to be introductions rather than real abstracts. This corresponded to a threshold of $57 \%$ novel unigrams.

For both OrangeSum Title and OrangeSum Abstract, we set aside 1500 pairs for testing, 1500 for validation, and used all the remaining ones for training. We make the dataset publicly available ${ }^{10}$. An example document with its summaries is provided in Table 4. More examples are available in appendix.

Analysis. Table 2 compares OrangeSum with XSum and the well-known CNN, DailyMail, and NY Times datasets. We can see that the two OrangeSum datasets are very similar to XSum in terms of statistics, but is one order of magnitude smaller than XSum. However, the size of OrangeSum still allows for effective finetuning, as we later demonstrate in our experiments.

Table 3 provides empirical evidence showing that like XSum, OrangeSum is less biased towards

\footnotetext{
${ }^{10} \mathrm{https} / / /$ github.com/Tixierae/OrangeSum
} 


\begin{tabular}{|c|c|c|}
\hline & Document & $\begin{array}{l}\text { Le } 18 \text { octobre dernier, Jacline Mouraud se faisait connaître en publiant sur Facebook une vidéo } \\
\text { dans laquelle elle poussait un "coup de gueule" contre le gouvernement. Aujourd'hui, la Bretonne } \\
\text { a pris ses distances par rapport au mouvement, notamment face à d'autres figures plus radicales } \\
\text { comme Éric Drouet. Jacline Mouraud réfléchit désormais à créer son propre parti, "la seule } \\
\text { chose envisageable", comme elle l'explique au JDD. Nicolas Sarkozy, "le seul qui a des couilles". } \\
\text { Cette figure des "gilets jaunes", accusée de faire le jeu de LREM estime que "le problème" } \\
\text { d'Emmanuel Macron "c'est qu'il est jeune". "Il devrait y avoir un âge minimum pour être } \\
\text { président : } 50 \text { ans", souligne Jacline Mouraud. Dans le JDD, elle raconte d'ailleurs avoir voté } \\
\text { blanc lors de la dernière présidentielle. En } 2007 \text { et 2012, c'est Nicolas Sarkozy, "le seul qui a des } \\
\text { couilles", que la figure des "gilets jaunes" avait soutenu. En attendant de se lancer, pas question } \\
\text { pour elle en tous les cas d'être candidate aux européennes sur une liste de La République en } \\
\text { marche. }\end{array}$ \\
\hline & mBARThez & $\begin{array}{l}\text { L'une des figures du mouvement ne sera toutefois pas candidate aux prochaines élections } \\
\text { européennes. } \\
\text { Jacline Mouraud, figure des “gilets jaunes", estime que le président d'Emmanuel Macron est } \\
\text { trop jeune pour être président. } \\
\text { Dans un entretien au JDD, la figure des "gilets jaunes" Jacline Mouraud révèle qu'elle réfléchit à } \\
\text { créer son propre parti. } \\
\text { Dans les colonnes du JDD, la figure des "gilets jaunes" explique qu'elle envisage de se présenter } \\
\text { aux européennes sur une liste La République en marche. } \\
\text { Retirée de la vie politique depuis plusieurs mois, Bretone Mouraud envisage de se lancer en } \\
\text { politique. Et elle réfléchit à quelque chose de plus, rapporte le JDD. }\end{array}$ \\
\hline & $\begin{array}{r}\text { Gold } \\
\text { mBART } \\
\text { mBARThez } \\
\text { BARThez } \\
\text { C2C }\end{array}$ & $\begin{array}{l}\text { "Gilets jaunes" : Jacline Mouraud réfléchit à créer son parti } \\
\text { "Gilets jaunes": Jacline Mouraud lance son propre parti } \\
\text { "Gilets jaunes": Jacline Mouraud prend ses distances } \\
\text { La figure des "gilets jaunes" Jacline Mouraud va créer son propre parti } \\
\text { "Gilets jaunes" : Jacline Mouraud réfléchit à sa propre candidature }\end{array}$ \\
\hline
\end{tabular}

Table 4: Doc 19233 from OrangeSum's test set, and associated summaries. Incorrect information in orange. C2C stands for CamemBERT2CamemBERT.

extractive systems compared with the traditional datasets used for abstractive summarization. There are $30 \%$ novel unigrams in the OrangeSum Abstract reference summaries and $26.5 \%$ in OrangeSum Title, compared with $35.7 \%$ in Xsum, $17 \%$ in CNN, $17 \%$ in DailyMail, and $23 \%$ in NY Times. This indicates that XSum and OrangeSum summaries are more abstractive. These observations are also confirmed by the fact that the two extractive baselines LEAD and EXT-ORACLE perform much more poorly on XSum and OrangeSum than on the other datasets.

\section{Experiments}

We compare BARThez and mBARThez with the following models, summarized in Table 5.

- mBART. The multilingual BART LARGE described in section 4.

- CamemBERT2CamemBERT (C2C). Following BERT2BERT (Rothe et al., 2020), we finetuned a sequence-to-sequence model whose both encoder and decoder parameters were initialized with CamemBERT LARGE weights. The only weights that were initialized randomly were the encoder-decoder attention weights.

- BART-random. Same architecture and vocabulary as BARThez, trained from scratch on the downstream tasks.

\subsection{Summarization}

All pretrained models were finetuned for 30 epochs and we used a learning rate that warmed up to 0.0001 ( $6 \%$ of the training steps) and then decreased linearly to 0 . BART-random was trained for 60 epochs. We selected the checkpoint associated with the best validation score to generate the test set summaries, using beam-search with a beam size of 4.

We report ROUGE-1-2-L scores (Lin, 2004) and BERTScore scores (Zhang et al., 2020) in Table 6.

Following Narayan et al. (2018), we included two extractive baselines, LEAD and EXTORACLE, which respectively extract the first sentence and the set of sentences maximizing ROUGE$\mathrm{L}$ from the document.

Quantitative results. Table 6 compares the performance of the models finetuned on the summarization task. While having four times less parameters, BARThez is on par with mBART, both in terms of ROUGE and BERTScore.

mBARThez provides a significant boost over BARThez and mBART and reaches best performance everywhere. This highlights the importance of adapting a multilingual pretrained model 


\begin{tabular}{|c|c|c|c|c|c|c|c|}
\hline & & Layers & Params & Vocab. size & Pretraining hours & Pretraining GPUs & Corpus size \\
\hline w & BART-random & 12 & 165 & 50 & 0 & NA & NA \\
\hline 㐫 & BARThez (ours) & 12 & 165 & 50 & 60 & 128 & 66 \\
\hline m & $\mathrm{C} 2 \mathrm{C}$ & 24 & 274 & 32 & 24 & 256 & 138 \\
\hline 幽 & mBART & 24 & 610 & 250 & 432 & 256 & 1369 \\
\hline 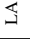 & mBARThez (ours) & 24 & 458 & 101 & 30 & $256+128$ & $1369+66$ \\
\hline
\end{tabular}

Table 5: Summary of the models used in our experiments. Parameters are given in millions, vocab sizes in thousands, and corpus sizes in GB. C2C stands for CamemBERT2CamemBERT.

\begin{tabular}{|c|c|c|c|c|c|c|c|c|c|}
\hline & \multicolumn{4}{|c|}{ Abstract } & \multicolumn{4}{|c|}{ Title } \\
\hline & & $\mathrm{R}-1$ & $\mathrm{R}-2$ & R-L & BertScore & $\mathrm{R}-1$ & $\mathrm{R}-2$ & R-L & BertScore \\
\hline & LEAD & 22.21 & 07.00 & 15.48 & $14.66 / 68.02$ & 19.84 & 08.11 & 16.13 & $15.75 / 68.43$ \\
\hline & EXT-ORACLE & 38.36 & 20.87 & 31.08 & $28.99 / 73.39$ & 31.62 & 17.06 & 28.26 & $25.15 / 71.95$ \\
\hline w & BART-random & 27.67 & 08.23 & 18.50 & $22.53 / 70.97$ & 28.76 & 13.15 & 25.20 & $29.67 / 73.65$ \\
\hline$\stackrel{4}{m}$ & BARThez (ours) & 31.44 & 12.77 & 22.23 & 27.51/72.84 & 40.86 & 23.68 & 36.03 & 40.61/77.74 \\
\hline & CamemBERT2CamemBERT & 29.23 & 09.79 & 19.95 & $25.53 / 72.09$ & 34.92 & 18.04 & 30.83 & $36.40 / 76.17$ \\
\hline$\tilde{\Xi}$ & mBART & 31.85 & 13.10 & 22.35 & $27.80 / 72.94$ & 40.74 & 23.70 & 36.04 & $40.42 / 77.67$ \\
\hline 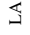 & mBARThez (ours) & 32.67 & 13.73 & 23.18 & 28.80/73.32 & 41.08 & 24.11 & 36.41 & 41.42/78.05 \\
\hline
\end{tabular}

Table 6: Results on OrangeSum. The two BertScore scores are with/without rescaling (Zhang et al., 2020).

to a specific language before finetuning (languageadaptive pretraining). This also suggests that, when proper adaptation is conducted, it can be advantageous to capitalize on a multilingual model to perform monolingual downstream tasks, probably because there are some translingual features and patterns to be learned.

Finally, all BART-based models outperform CamemBERT2CamemBERT by a significant margin.

Human evaluation. To validate our positive quantitative results, we conducted a human evaluation study with 11 French native speakers. The evaluators were $\mathrm{PhD}$ students from the computer science department of our university, working in NLP and other fields of AI. They volunteered after receiving an email announcement. Following Narayan et al. (2018), we used Best-Worst Scaling (Louviere et al., 2015). Two summaries from two different systems, along with their input document, were presented to a human annotator who had to decide which one was better. We asked evaluators to base their judgments on accuracy (does the summary contain accurate facts?), informativeness (is important information captured?) and fluency (is the summary written in well-formed French?).

We randomly sampled 14 documents from the test set of OrangeSum Abstract, and for each document, generated all possible pairs of BARThez, mBARThez, mBART, C2C, and GT summaries, resulting in 140 pairs. Each pair was randomly assigned to three different annotators, resulting in 420 evaluation tasks in total. The final score of a model was given as the percentage of time its summary was chosen as best minus the percentage of time it was chosen as worst. Scores are reported in Table 10. mBARThez reaches first place, like for the quantitative results, but with an even wider margin. It is also interesting to note that BARThez, which was on par with mBART quantitatively, significantly outperforms it terms of human evaluations. The negative score of CamemBERT2CamemBERT means that its summaries were judged to be worse more often than not.

Suprisingly, BARThez and mBARThez' summaries were often judged better than the ground truth ones. This could be explained by the fact that the GT summaries, while well-written, amy contain information that is missing from the documents, such as dates. Annotators may consider such information as inaccurate (model hallucinations).

Qualitative results. As shown in Table 7, mBARThez is more abstractive than BARThez and mBART. E.g., mBARThez introduces on average $15.48 \%$ of novel unigrams in its summaries for the Abstract task, compared with 10.93 and 13.40 for BARThez and mBART, respectively. It is interesting to note that despite this superior abstractivity, mBARThez still reaches first place everywhere in terms of the ROUGE metric, which measures ngram overlap. We hypothesize that BARThez is less abstractive than mBART and mBARThez because it has four times less parameters (BASE vs LARGE architecture).

Finally, it is also to be noted that CamemBERT2CamemBERT (C2C) introduces many new words, which could be considered a good thing at first. However, it also repeats itself a lot (see Table 9) and has low ROUGE, BERTSum, and human 


\begin{tabular}{|c|c|c|c|c|c|c|c|c|c|}
\hline & \multicolumn{4}{|c|}{ OrangeSum Abstract } & \multicolumn{4}{|c|}{ OrangeSum Title } \\
\hline & & 1-grams & 2-grams & 3-grams & 4-grams & 1-grams & 2-grams & 3-grams & 4-grams \\
\hline & Gold & 30.03 & 67.15 & 81.94 & 88.30 & 26.54 & 66.70 & 84.18 & 91.12 \\
\hline BASE & BARThez (ours) & 10.93 & 34.03 & 47.97 & 56.80 & 16.69 & 51.70 & 72.05 & 82.49 \\
\hline \multirow{3}{*}{$\begin{array}{l}\text { 멍 } \\
\text { 전 }\end{array}$} & $\mathrm{C} 2 \mathrm{C}$ & 39.43 & 79.12 & 92.04 & 96.28 & 33.82 & 75.74 & 91.77 & 96.71 \\
\hline & mBART & 13.40 & 38.94 & 53.69 & 62.61 & 16.89 & 52.28 & 73.12 & 82.74 \\
\hline & mBARThez (ours) & 15.48 & 43.34 & 58.53 & 67.30 & 17.79 & 53.41 & 73.38 & 82.94 \\
\hline
\end{tabular}

Table 7: Proportion of novel n-grams in the generated summaries. C2C stands for CamemBERT2CamemBERT. Note that C2C's high scores are misleading as many of the introduced words are irrelevant.

\begin{tabular}{|c|c|c|c|c|c|c|}
\hline & & CLS-books & CLS-DVD & CLS-music & PAWSX & XNLI \\
\hline \multirow{5}{*}{$\underset{\infty}{\mathbb{2}}$} & mBERT $^{\dagger}$ (Devlin et al., 2019) & 86.15 & 89.90 & 86.65 & 89.30 & 76.9 \\
\hline & CamemBERT $_{B A S E^{\dagger}}$ (Martin et al., 2020) & 92.30 & 93.00 & 94.85 & 90.14 & 81.20 \\
\hline & FlauBERT $_{B A S E}^{\dagger}$ (Le et al., 2020) & 92.30 & 92.45 & 94.10 & 89.49 & 80.60 \\
\hline & BARThez (ours) & 94.47 0.17 & 93.17 0.40 & 94.97 0.25 & $88.90_{0.24}$ & $80.73_{0.40}$ \\
\hline & BART-random & $76.37_{0.34}$ & $73.20_{0.65}$ & $76.00_{1.28}$ & $55.27_{0.33}$ & $60.43_{0.87}$ \\
\hline \multirow{4}{*}{ 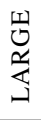 } & Camembert $_{L A R G E}$ (Martin et al., 2020) & $\mathbf{9 5 . 4 7} 0.33$ & $\mathbf{9 5 . 3 7} 0.07$ & $\mathbf{9 6 . 0 0 _ { 0 . 2 9 }}$ & $\mathbf{9 1 . 8 3} 0.54$ & $\mathbf{8 5 . 3 3} 0.05$ \\
\hline & Flaubert $_{L A R G E}^{\dagger}$ (Le et al., 2020) & 95.00 & 94.10 & 95.85 & 89.34 & 83.40 \\
\hline & mBART (Liu et al., 2020) & $93.40_{0.22}$ & $93.10_{0.20}$ & $93.13_{0.79}$ & $89.70_{0.22}$ & $81.07_{0.38}$ \\
\hline & mBARThez (ours) & $94.63_{0.05}$ & 94.030 .09 & $95.30_{0.16}$ & $90.90_{0.22}$ & $81.87_{0.50}$ \\
\hline
\end{tabular}

Table 8: Accuracy on discriminative tasks (standard deviation as subscript). ${ }^{\dagger}$ are taken from Le et al. (2020).

\begin{tabular}{|c|c|c|c|}
\hline & & Length & Repetitions (\%) \\
\hline \multirow{5}{*}{ 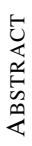 } & Gold & 32.12 & 11.47 \\
\hline & mBART & 28.20 & 7.47 \\
\hline & mBARThez & 29.45 & 8.60 \\
\hline & BARThez & 29.10 & 14.47 \\
\hline & $\mathrm{C} 2 \mathrm{C}$ & 30.68 & 23.00 \\
\hline \multirow{5}{*}{$\stackrel{\text { I }}{\mathrm{E}}$} & Gold & 11.42 & 0.93 \\
\hline & mBART & 10.79 & 1.73 \\
\hline & mBARThez & 11.03 & 2.27 \\
\hline & BARThez & 11.19 & 2.73 \\
\hline & $\mathrm{C} 2 \mathrm{C}$ & 11.23 & 19.53 \\
\hline
\end{tabular}

Table 9: Summary statistics.

\begin{tabular}{|cl|c|}
\hline \multicolumn{2}{|c|}{ System } & Score \\
\hline & Gold & 14.29 \\
\hline \hline BASE & BARThez (ours) & $\mathbf{2 1 . 4 3}$ \\
\hline 먼 & CamemBERT2CamemBERT & -75.00 \\
mBART & 11.90 \\
mBARThez (ours) & $\mathbf{2 7 . 3 8}$ \\
\hline
\end{tabular}

Table 10: Human evaluation using Best-Worst Scaling.

evaluation scores. A manual observation revealed that actually, many of the new words introduced by $\mathrm{C} 2 \mathrm{C}$ are irrelevant (see summary examples in appendix).

Also, like in Rothe et al. (2020), we computed the length of the summaries, and the percentage of summaries with at least one non-stopword repetition. We used as stopwords the 500 most frequent words from the system and gold summaries, across all documents. As can be seen in Table 9, for both the Abstract and Title tasks, all models generated summaries of sizes very close to that of the Gold summaries.

In terms of repetitions, the less redundant models, closest to the ground truth, are mBART and mBARThez. This is especially apparent on the Abstract task, where potential for repetition is greater.
On this task, mBART and mBARThez show less than $9 \%$ repetitions, compared with 14.5 and 23 for BARThez and $\mathrm{C} 2 \mathrm{C}$ (resp.), and 11.5 in the references. $\mathrm{C} 2 \mathrm{C}$ is also way more redundant than the other models and far from the reference on the Title task, with $19.5 \%$ of repetitions.

\subsection{Discriminative tasks}

In addition to generative tasks, BART-like models can perform discriminative tasks (Lewis et al., 2020). In the case of sequence classification, the input sequence is fed to both the encoder and the decoder, and the representation of the last token in the sequence is used by adding a classification head on top of it. When the input consists of several sentences, these sentences are separated with a special token and pasted together. We evaluate the different models on five discriminative tasks from the FLUE benchmark ${ }^{11}$ (Le et al., 2020), the French equivalent of GLUE (Wang et al., 2019b).

- CLS. The Cross-lingual Sentiment analysis dataset (Prettenhofer and Stein, 2010) is made of Amazon reviews to be classified as positive or negative. It contains 3 product categories: books, DVD and music. The train and test sets are balanced and contain 2000 examples (each) per product category. Following Le et al. (2020), we used $20 \%$ of the train set as validation set.

- PAWSX. The Cross-lingual Adversarial Dataset for Paraphrase Identification (Yang et al., 2019) contains pairs of sentences, and the task is to predict whether they are semantically equivalent.

\footnotetext{
$11_{\text {https://github.com/getalp/Flaubert/tree/master/flue }}$
} 
There are 49401 examples for training, 1992 for development, and 1985 for testing.

- XNLI. The Cross-lingual NLI corpus (Conneau et al., 2018) contains pairs of sentences, and the task is to predict whether the first one (premise) entails the second one (hypothesis), contradicts it, or neither entails nor contradicts it (neutral relationship). 392702 pairs are used for training, 2490 for development, and 5010 for testing.

Training details. In all experiments, we finetuned the model for 10 epochs with a learning rate chosen from $\left\{10^{-4}, 5.10^{-5}, 10^{-5}\right\}$ based on the best validation score. We repeated each experiment 3 times with different seeds and report the mean and standard deviation.

Results. As shown in Table 8, among the models having a BASE architecture, BARThez is best in the three sentiment analysis tasks, while being very close to CamemBERT and FlauBERT in the paraphrasing and inference tasks.

Among the LARGE models, mBARThez outperforms mBART in all tasks, showing again the importance of language-adaptive pretraining. On the other hand, CamemBERT and FlauBERT outperform mBARThez in most of the tasks, which could be attributed to the fact that CamemBERT and FlauBERT were trained for approximately 10 times more GPU hours on a monolingual French corpus. Nevertheless, Given that huge difference in monolingual training time, it is remarkable that mBARThez is so close, and sometimes outperforms, FlauBERT, with e.g., a 1.56 margin on PAWSX.

We can conclude that the ability of BARThez and mBARThez to perform well on generative tasks does not appear to come at the expense of a decrease in performance on discriminative tasks, which is in line with the results presented in the BART paper (Lewis et al., 2020).

\section{Conclusion}

We released BARThez and mBARThez, the first French large-scale pretrained seq2seq models, as well as a novel French summarization dataset, OrangeSum, inspired by the XSum dataset. We showed our proposed models to be very competitive with the state of the art on generative and discriminative tasks. By evaluating our models on the summarization dataset we showed that: (1) BARThez is on par with mBART while having four times less parameters, and that (2) mBARThez pro- vides a significant boost over mBART by simply adding a relatively affordable language-adaptive phase to the pretraining. In addition, we evaluated BARThez and mBARThez on 5 sentiment analysis, paraphrasing, and natural language inference tasks against cutting edge BERT-based French language models (FlauBERT and CamemBERT), and obtained very competitive results. An interesting area for future work is to further explore languageadaptive pretraining.

\section{Acknowledgments}

We are thankful to the National Center for Scientific Research (CNRS) for giving us access to their Jean Zay supercomputer, under allocation 2020AD011011499. Finally, We thank the three anonymous reviewers for their constructive feedback.

\section{References}

Wissam Antoun, Fady Baly, and Hazem Hajj. 2020. AraBERT: Transformer-based model for Arabic language understanding. In Proceedings of the 4th Workshop on Open-Source Arabic Corpora and Processing Tools, with a Shared Task on Offensive Language Detection, pages 9-15, Marseille, France. European Language Resource Association.

ATILF and CLLE. 2020. Corpus journalistique issu de l'est républicain. ORTOLANG (Open Resources and TOols for LANGuage) -www.ortolang.fr.

Piotr Bojanowski, Edouard Grave, Armand Joulin, and Tomas Mikolov. 2017. Enriching word vectors with subword information. Transactions of the Association for Computational Linguistics, 5:135-146.

José Cañete, Gabriel Chaperon, Rodrigo Fuentes, and Jorge Pérez. 2020. Spanish pre-trained bert model and evaluation data. In to appear in PMLADC at ICLR 2020.

Alexis Conneau, Kartikay Khandelwal, Naman Goyal, Vishrav Chaudhary, Guillaume Wenzek, Francisco Guzmán, Edouard Grave, Myle Ott, Luke Zettlemoyer, and Veselin Stoyanov. 2020. Unsupervised cross-lingual representation learning at scale. In Proceedings of the 58th Annual Meeting of the Association for Computational Linguistics, pages 84408451, Online. Association for Computational Linguistics.

Alexis Conneau and Guillaume Lample. 2019. Crosslingual language model pretraining. In Advances in Neural Information Processing Systems 32: Annual Conference on Neural Information Processing Systems 2019, NeurIPS 2019, December 8-14, 2019, Vancouver, BC, Canada, pages 7057-7067. 
Alexis Conneau, Ruty Rinott, Guillaume Lample, Adina Williams, Samuel Bowman, Holger Schwenk, and Veselin Stoyanov. 2018. XNLI: Evaluating cross-lingual sentence representations. In Proceedings of the 2018 Conference on Empirical Methods in Natural Language Processing, pages 2475-2485, Brussels, Belgium. Association for Computational Linguistics.

Wietse de Vries, Andreas van Cranenburgh, Arianna Bisazza, Tommaso Caselli, Gertjan van Noord, and Malvina Nissim. 2019. Bertje: A dutch bert model. ArXiv preprint, abs/1912.09582.

Pieter Delobelle, Thomas Winters, and Bettina Berendt. 2020. RobBERT: a Dutch RoBERTa-based Language Model. In Findings of the Association for Computational Linguistics: EMNLP 2020, pages 3255-3265, Online. Association for Computational Linguistics.

Jacob Devlin, Ming-Wei Chang, Kenton Lee, and Kristina Toutanova. 2019. BERT: Pre-training of deep bidirectional transformers for language understanding. In Proceedings of the 2019 Conference of the North American Chapter of the Association for Computational Linguistics: Human Language Technologies, Volume 1 (Long and Short Papers), pages 4171-4186, Minneapolis, Minnesota. Association for Computational Linguistics.

Andreas Eisele and Yu Chen. 2010. MultiUN: A multilingual corpus from united nation documents. In Proceedings of the Seventh International Conference on Language Resources and Evaluation (LREC'10), Valletta, Malta. European Language Resources Association (ELRA).

Suchin Gururangan, Ana Marasović, Swabha Swayamdipta, Kyle Lo, Iz Beltagy, Doug Downey, and Noah A. Smith. 2020. Don't stop pretraining: Adapt language models to domains and tasks. In Proceedings of the 58th Annual Meeting of the Association for Computational Linguistics, pages 8342-8360, Online. Association for Computational Linguistics.

Karl Moritz Hermann, Tomás Kociský, Edward Grefenstette, Lasse Espeholt, Will Kay, Mustafa Suleyman, and Phil Blunsom. 2015. Teaching machines to read and comprehend. In Advances in Neural Information Processing Systems 28: Annual Conference on Neural Information Processing Systems 2015, December 7-12, 2015, Montreal, Quebec, Canada, pages $1693-1701$.

Jeremy Howard and Sebastian Ruder. 2018. Universal language model fine-tuning for text classification. In Proceedings of the 56th Annual Meeting of the Association for Computational Linguistics (Volume 1: Long Papers), pages 328-339, Melbourne, Australia. Association for Computational Linguistics.

Diederik P. Kingma and Jimmy Ba. 2015. Adam: A method for stochastic optimization. In 3rd International Conference on Learning Representations,
ICLR 2015, San Diego, CA, USA, May 7-9, 2015, Conference Track Proceedings.

Alex Krizhevsky, Ilya Sutskever, and Geoffrey E. Hinton. 2012. Imagenet classification with deep convolutional neural networks. In Advances in Neural Information Processing Systems 25: 26th Annual Conference on Neural Information Processing Systems 2012. Proceedings of a meeting held December 36, 2012, Lake Tahoe, Nevada, United States, pages 1106-1114.

Taku Kudo and John Richardson. 2018. SentencePiece: A simple and language independent subword tokenizer and detokenizer for neural text processing. In Proceedings of the 2018 Conference on Empirical Methods in Natural Language Processing: System Demonstrations, pages 66-71, Brussels, Belgium. Association for Computational Linguistics.

Yuri Kuratov and Mikhail Arkhipov. 2019. Adaptation of deep bidirectional multilingual transformers for russian language. ArXiv preprint, abs/1905.07213.

Hang Le, Loïc Vial, Jibril Frej, Vincent Segonne, Maximin Coavoux, Benjamin Lecouteux, Alexandre Allauzen, Benoit Crabbé, Laurent Besacier, and Didier Schwab. 2020. FlauBERT: Unsupervised language model pre-training for French. In Proceedings of the 12th Language Resources and Evaluation Conference, pages 2479-2490, Marseille, France. European Language Resources Association.

Mike Lewis, Yinhan Liu, Naman Goyal, Marjan Ghazvininejad, Abdelrahman Mohamed, Omer Levy, Veselin Stoyanov, and Luke Zettlemoyer. 2020. BART: Denoising sequence-to-sequence pretraining for natural language generation, translation, and comprehension. In Proceedings of the 58th Annual Meeting of the Association for Computational Linguistics, pages 7871-7880, Online. Association for Computational Linguistics.

Xian Li, Paul Michel, Antonios Anastasopoulos, Yonatan Belinkov, Nadir Durrani, Orhan Firat, Philipp Koehn, Graham Neubig, Juan Pino, and Hassan Sajjad. 2019. Findings of the first shared task on machine translation robustness. In Proceedings of the Fourth Conference on Machine Translation (Volume 2: Shared Task Papers, Day 1), pages 91-102, Florence, Italy. Association for Computational Linguistics.

Chin-Yew Lin. 2004. ROUGE: A package for automatic evaluation of summaries. In Text Summarization Branches Out, pages 74-81, Barcelona, Spain. Association for Computational Linguistics.

Yang Liu and Mirella Lapata. 2019. Text summarization with pretrained encoders. In Proceedings of the 2019 Conference on Empirical Methods in Natural Language Processing and the 9th International Joint Conference on Natural Language Processing (EMNLP-IJCNLP), pages 3730-3740, Hong Kong, China. Association for Computational Linguistics. 
Yinhan Liu, Jiatao Gu, Naman Goyal, Xian Li, Sergey Edunov, Marjan Ghazvininejad, Mike Lewis, and Luke Zettlemoyer. 2020. Multilingual denoising pre-training for neural machine translation. Transactions of the Association for Computational Linguistics, 8:726-742.

Yinhan Liu, Myle Ott, Naman Goyal, Jingfei Du, Mandar Joshi, Danqi Chen, Omer Levy, Mike Lewis, Luke Zettlemoyer, and Veselin Stoyanov. 2019. Roberta: A robustly optimized bert pretraining approach. ArXiv preprint, abs/1907.11692.

Jordan J Louviere, Terry N Flynn, and Anthony Alfred John Marley. 2015. Best-worst scaling: Theory, methods and applications. Cambridge University Press.

Louis Martin, Benjamin Muller, Pedro Javier Ortiz Suárez, Yoann Dupont, Laurent Romary, Éric de la Clergerie, Djamé Seddah, and Benoît Sagot. 2020. CamemBERT: a tasty French language model. In Proceedings of the 58th Annual Meeting of the Association for Computational Linguistics, pages 7203-7219, Online. Association for Computational Linguistics.

Tomás Mikolov, Ilya Sutskever, Kai Chen, Gregory S. Corrado, and Jeffrey Dean. 2013. Distributed representations of words and phrases and their compositionality. In Advances in Neural Information Processing Systems 26: 27th Annual Conference on Neural Information Processing Systems 2013. Proceedings of a meeting held December 5-8, 2013, Lake Tahoe, Nevada, United States, pages 31113119.

Shashi Narayan, Shay B. Cohen, and Mirella Lapata. 2018. Don't give me the details, just the summary! topic-aware convolutional neural networks for extreme summarization. In Proceedings of the 2018 Conference on Empirical Methods in Natural Language Processing, pages 1797-1807, Brussels, Belgium. Association for Computational Linguistics.

Myle Ott, Sergey Edunov, Alexei Baevski, Angela Fan, Sam Gross, Nathan Ng, David Grangier, and Michael Auli. 2019. fairseq: A fast, extensible toolkit for sequence modeling. In Proceedings of the 2019 Conference of the North American Chapter of the Association for Computational Linguistics (Demonstrations), pages 48-53, Minneapolis, Minnesota. Association for Computational Linguistics.

Jeffrey Pennington, Richard Socher, and Christopher Manning. 2014. GloVe: Global vectors for word representation. In Proceedings of the 2014 Conference on Empirical Methods in Natural Language Processing (EMNLP), pages 1532-1543, Doha, Qatar. Association for Computational Linguistics.

Matthew E. Peters, Mark Neumann, Mohit Iyyer, Matt Gardner, Christopher Clark, Kenton Lee, and Luke
Zettlemoyer. 2018. Deep contextualized word representations. In Proceedings of the 2018 Conference of the North American Chapter of the Association for Computational Linguistics: Human Language Technologies, Volume 1 (Long Papers), pages 2227-2237, New Orleans, Louisiana. Association for Computational Linguistics.

Marco Polignano, Pierpaolo Basile, Marco de Gemmis, Giovanni Semeraro, and Valerio Basile. 2019. Alberto: Italian bert language understanding model for nlp challenging tasks based on tweets. In CLiC-it.

Peter Prettenhofer and Benno Stein. 2010. Crosslanguage text classification using structural correspondence learning. In Proceedings of the 48th Annual Meeting of the Association for Computational Linguistics, pages 1118-1127, Uppsala, Sweden. Association for Computational Linguistics.

Weizhen Qi, Yu Yan, Yeyun Gong, Dayiheng Liu, Nan Duan, Jiusheng Chen, Ruofei Zhang, and Ming Zhou. 2020. ProphetNet: Predicting future n-gram for sequence-to-SequencePre-training. In Findings of the Association for Computational Linguistics: EMNLP 2020, pages 2401-2410, Online. Association for Computational Linguistics.

Alec Radford, Jeffrey Wu, Rewon Child, David Luan, Dario Amodei, and Ilya Sutskever. 2019. Language models are unsupervised multitask learners. OpenAI blog, 1(8):9.

Anna Rogers, Olga Kovaleva, and Anna Rumshisky. 2020. A primer in BERTology: What we know about how BERT works. Transactions of the Association for Computational Linguistics, 8:842-866.

Sascha Rothe, Shashi Narayan, and Aliaksei Severyn. 2020. Leveraging pre-trained checkpoints for sequence generation tasks. Transactions of the Association for Computational Linguistics, 8:264-280.

Rico Sennrich, Barry Haddow, and Alexandra Birch. 2016. Neural machine translation of rare words with subword units. In Proceedings of the 54th Annual Meeting of the Association for Computational Linguistics (Volume 1: Long Papers), pages 17151725, Berlin, Germany. Association for Computational Linguistics.

Raivis Skadinš, Jörg Tiedemann, Roberts Rozis, and Daiga Deksne. 2014. Billions of parallel words for free: Building and using the EU bookshop corpus. In Proceedings of the Ninth International Conference on Language Resources and Evaluation (LREC'14), pages 1850-1855, Reykjavik, Iceland. European Language Resources Association (ELRA).

Kaitao Song, Xu Tan, Tao Qin, Jianfeng Lu, and TieYan Liu. 2019. MASS: masked sequence to sequence pre-training for language generation. In Proceedings of the 36th International Conference on Machine Learning, ICML 2019, 9-15 June 2019, 
Long Beach, California, USA, volume 97 of Proceedings of Machine Learning Research, pages 5926-5936. PMLR.

Fábio Souza, Rodrigo Nogueira, and Roberto Lotufo. 2019. Portuguese named entity recognition using bert-crf. ArXiv preprint, abs/1909.10649.

Jörg Tiedemann. 2012. Parallel data, tools and interfaces in OPUS. In Proceedings of the Eighth International Conference on Language Resources and Evaluation (LREC'12), pages 2214-2218, Istanbul, Turkey. European Language Resources Association (ELRA).

Ashish Vaswani, Noam Shazeer, Niki Parmar, Jakob Uszkoreit, Llion Jones, Aidan N. Gomez, Lukasz Kaiser, and Illia Polosukhin. 2017. Attention is all you need. In Advances in Neural Information Processing Systems 30: Annual Conference on Neural Information Processing Systems 2017, December 49, 2017, Long Beach, CA, USA, pages 5998-6008.

Alex Wang, Yada Pruksachatkun, Nikita Nangia, Amanpreet Singh, Julian Michael, Felix Hill, Omer Levy, and Samuel R. Bowman. 2019a. Superglue: A stickier benchmark for general-purpose language understanding systems. In Advances in Neural Information Processing Systems 32: Annual Conference on Neural Information Processing Systems 2019, NeurIPS 2019, December 8-14, 2019, Vancouver, $B C$, Canada, pages 3261-3275.

Alex Wang, Amanpreet Singh, Julian Michael, Felix Hill, Omer Levy, and Samuel R. Bowman. 2019b. GLUE: A multi-task benchmark and analysis platform for natural language understanding. In $7 \mathrm{th}$ International Conference on Learning Representations, ICLR 2019, New Orleans, LA, USA, May 6-9, 2019. OpenReview.net.

Yinfei Yang, Yuan Zhang, Chris Tar, and Jason Baldridge. 2019. PAWS-X: A cross-lingual adversarial dataset for paraphrase identification. In Proceedings of the 2019 Conference on Empirical Methods in Natural Language Processing and the 9th International Joint Conference on Natural Language Processing (EMNLP-IJCNLP), pages 36873692, Hong Kong, China. Association for Computational Linguistics.

Tianyi Zhang, Varsha Kishore, Felix Wu, Kilian Q. Weinberger, and Yoav Artzi. 2020. Bertscore: Evaluating text generation with BERT. In 8th International Conference on Learning Representations, ICLR 2020, Addis Ababa, Ethiopia, April 26-30, 2020. OpenReview.net. 


\section{Appendices}

In what follows, we provide, for 10 documents randomly selected from OrangeSum's test set, the reference and model summaries for each task (Abstract and Title).

\begin{tabular}{|c|c|c|}
\hline & Document & $\begin{array}{l}\text { "Nous pouvons confirmer à ce stade que cinq personnes ont péri. Au moins } 70 \text { personnes sont } \\
\text { dans un état inconscient dans un hôpital non loin, et dans l'ensemble, entre } 200 \text { et } 500 \text { personnes } \\
\text { reçoivent des soins", a déclaré Swaroop Rani, une responsable de la police de Visakhapatnam, } \\
\text { dans l'État de l'Andhra Pradesh.Opérée par LG Polymers, l'usine est située en bordure de la ville } \\
\text { industrielle et portuaire de Visakhapatnam. L'agglomération compte une population d'environ } \\
5 \text { millions de personnes.Le gaz "avait été laissé là à cause du confinement. Cela a mené à une } \\
\text { réaction chimique et de la chaleur est apparue dans les réservoirs, et le gaz a fui à cause de cela", } \\
\text { a expliqué Mme Rani. L'Inde est actuellement confinée depuis fin mars pour lutter contre la } \\
\text { pandémie de coronavirus."Nous avons reçu un appel d'urgence de villageois autour de 03H30 } \\
\text { du matin aujourd'hui (mercredi } 22 \mathrm{H} 00 \text { GMT, ndlr). Ils disaient qu'il y avait du gaz dans l'air", } \\
\text { a-t-elle poursuivi."Nous sommes arrivés immédiatement sur place. On pouvait sentir le gaz } \\
\text { dans l'air et il ne n'était possible à aucun d'entre nous de rester là plus de quelques minutes", } \\
\text { a-t-elle décrit.L'Inde a été le théâtre en décembre 1984 d'un des pires accidents industriels de } \\
\text { l'Histoire, lorsque } 40 \text { tonnes de gaz s'étaient échappées d'une usine de pesticides de la ville de } \\
\text { Bhopal (centre).Quelque } 3.500 \text { personnes avaient péri en quelques jours, principalement dans } \\
\text { des bidonvilles situés autour de cette usine d'Union Carbide, et des milliers d'autres dans les } \\
\text { années qui ont suivi. }\end{array}$ \\
\hline & mBARThez & $\begin{array}{l}\text { Au moins cinq personnes ont péri et plus d'un millier ont été hospitalisées à } \\
\text { la suite d'une fuite de gaz dans une usine chimique du sud-est de l'Inde, ont } \\
\text { annoncé jeudi la police et une source officielle. } \\
\text { Cinq personnes sont mortes mercredi dans l'accident d'une usine de pesticides } \\
\text { qui s'est produite en Inde, à la suite du confinement lié à l'épidémie de Covid-19, } \\
\text { ont indiqué des responsables. } \\
\text { Une explosion a fait cinq morts mercredi dans une usine de pesticides du centre } \\
\text { de l'Inde, après que le gaz a fui dans les réservoirs après une réaction chimique, } \\
\text { ont indiqué les autorités. } \\
\text { Une dizaine de personnes ont péri et des centaines d'autres ont été blessées } \\
\text { mercredi dans une usine de pesticides près de Visakhapatnam, dans le sud de } \\
\text { l'Inde, a annoncé la police. } \\
\text { Au moins vingt personnes sont mortes, dont cinq sont mortes et cinq sont portées } \\
\text { disparues, selon un bilan officiel lundi après-midi en Inde, faisant craindre une } \\
\text { fuite de gaz meurtrière dans le pays, selon une source gouvernementale à l'AFP. }\end{array}$ \\
\hline & $\begin{array}{r}\text { Gold } \\
\text { mBART } \\
\text { mBARThez } \\
\text { BARThez } \\
\text { C2C }\end{array}$ & $\begin{array}{l}\text { Fuite de gaz dans une usine en Inde: } 5 \text { morts, au moins } 1.000 \text { personnes } \\
\text { hospitalisées } \\
\text { Inde: cinq morts dans un accident de la usine de pesticides } \\
\text { Inde: au moins cinq morts dans le crash d'une usine de pesticides } \\
\text { Inde: cinq morts dans un glissement de terrain à Visakhapatnam } \\
\text { Inde: cinq morts dans un gaz mortel dans un usine de recyclage }\end{array}$ \\
\hline
\end{tabular}

Table 11: C2C stands for CamemBERT2CamemBERT. OrangeSum document 12158. 


\begin{tabular}{|c|c|c|}
\hline & Document & 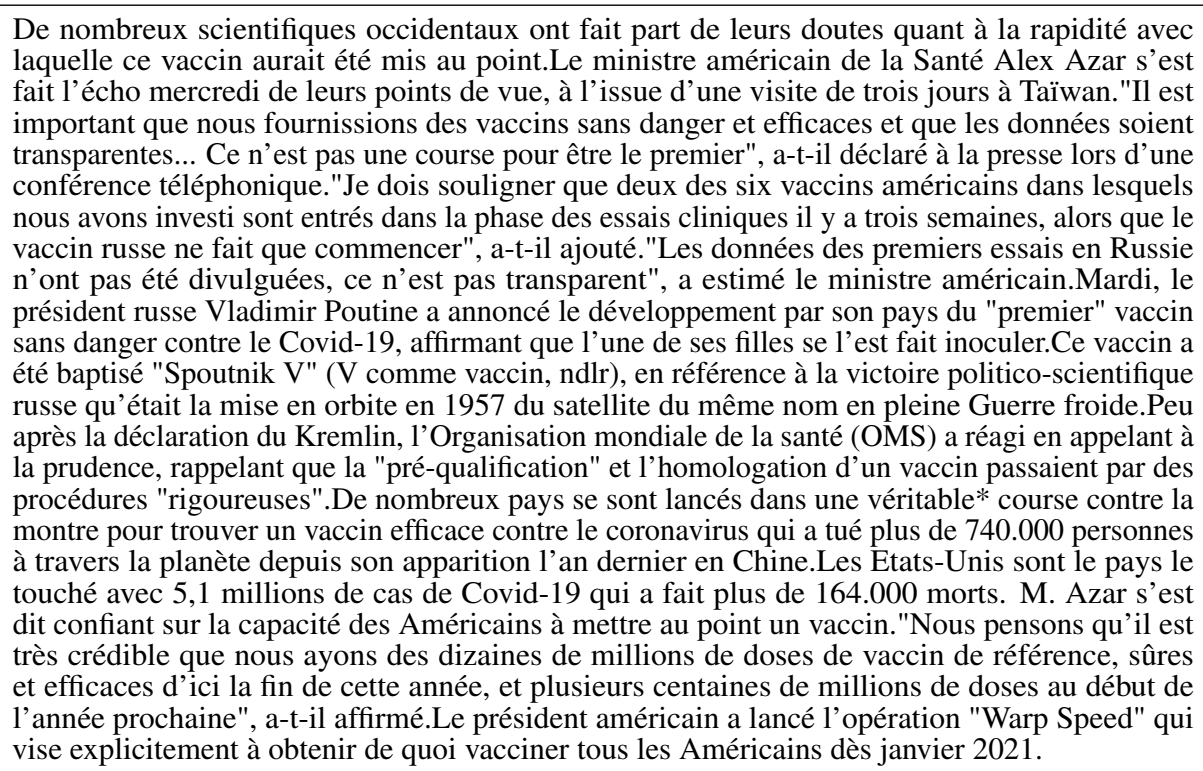 \\
\hline & mBARThez & $\begin{array}{l}\text { Le ministre américain de la Santé a fait part mercredi de son scepticisme } \\
\text { après l'annonce du développement par la Russie du "premier" vaccin contre le } \\
\text { coronavirus assurant une "immunité durable". } \\
\text { Le ministre américain de la Santé s'est exprimé mercredi sur la possibilité d'un } \\
\text { vaccin efficace contre le coronavirus. } \\
\text { Le ministre de la Santé américain Alex Azar a déclaré mercredi que les Etats- } \\
\text { Unis et la Russie ont fait des essais cliniques d'un vaccin contre le coronavirus } \\
\text { "sans danger", alors que le président russe Vladimir Poutine a déclaré mardi } \\
\text { avoir inoculé une de ses filles. } \\
\text { Les Etats-Unis sont le pays le plus touché par la pandémie de coronavirus après } \\
\text { la Russie qui a pourtant annoncé avoir mis au point le "premier vaccin", a estimé } \\
\text { le ministre américain de la Santé. } \\
\text { Le ministre américain de la Santé a souligné que la Russie avait développé des } \\
\text { capacités capables de détecter et de tester si le pays n'était pas vaccin contre } \\
\text { le nouveau coronavirus, mais a jugé prudent "dans l'attente de la publication } \\
\text { d'une réponse scientifique", }\end{array}$ \\
\hline & $\begin{array}{r}\text { Gold } \\
\text { mBART } \\
\text { mBARThez } \\
\text { BARThez }\end{array}$ & $\begin{array}{l}\text { Coronavirus: le ministre américain de la Santé sceptique au sujet du vaccin } \\
\text { russe } \\
\text { Vaccin " sans danger": les Américains investis en Russie, selon Alex Azar } \\
\text { Vaccin russe: les Américains appelés à la prudence } \\
\text { Un vaccin russe contre le Covid-19 en vue aux Etats-Unis, selon le ministre } \\
\text { américain de la Santé } \\
\text { Coronavirus: les Etats-Unis pas en "cours de combattant" face à un vaccin } \\
\text { expérimental }\end{array}$ \\
\hline
\end{tabular}

Table 12: C2C stands for CamemBERT2CamemBERT. OrangeSum document 33555. 


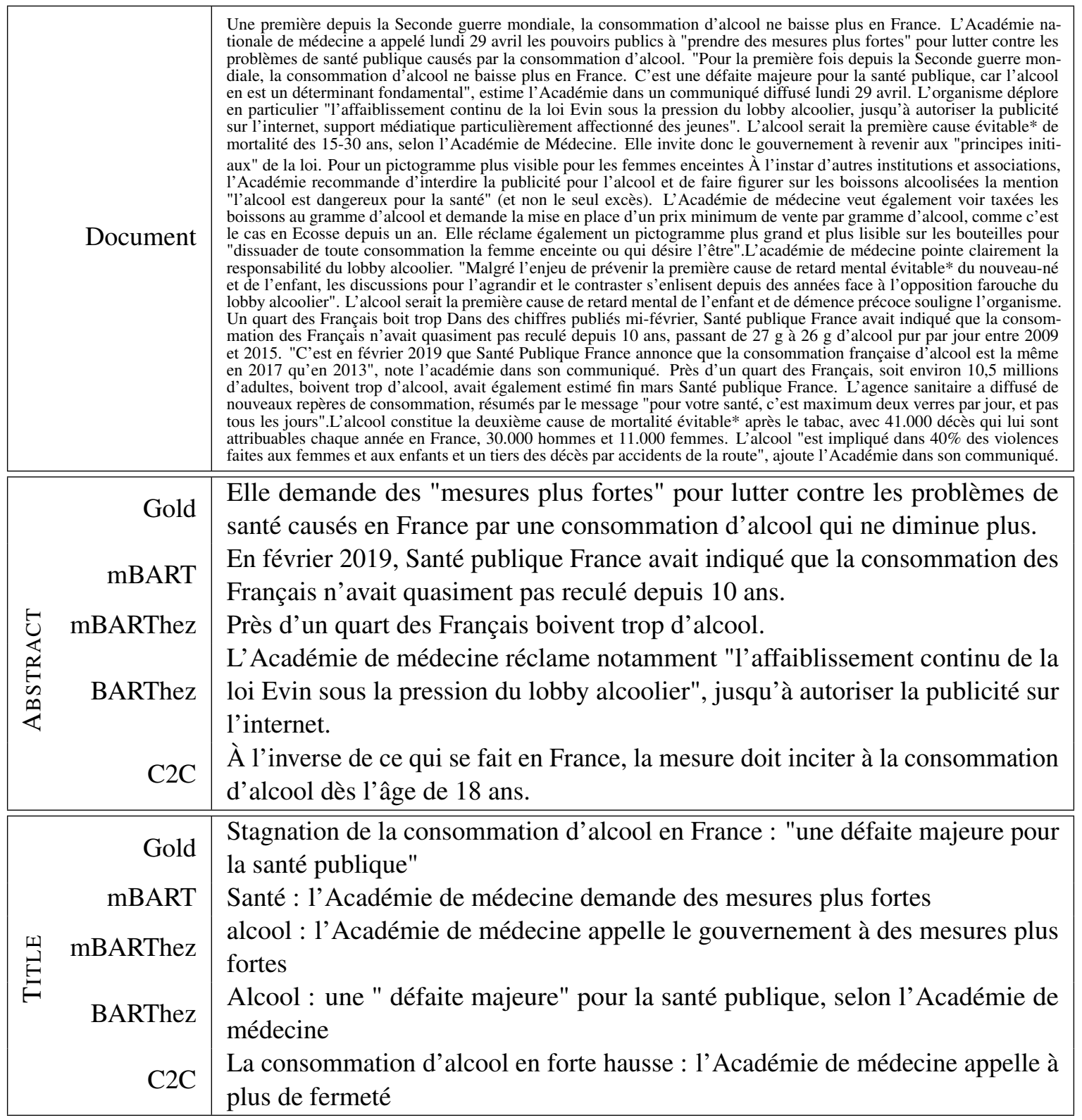

Table 13: C2C stands for CamemBERT2CamemBERT. OrangeSum document 25148. 


\begin{tabular}{|c|c|c|}
\hline & Document & 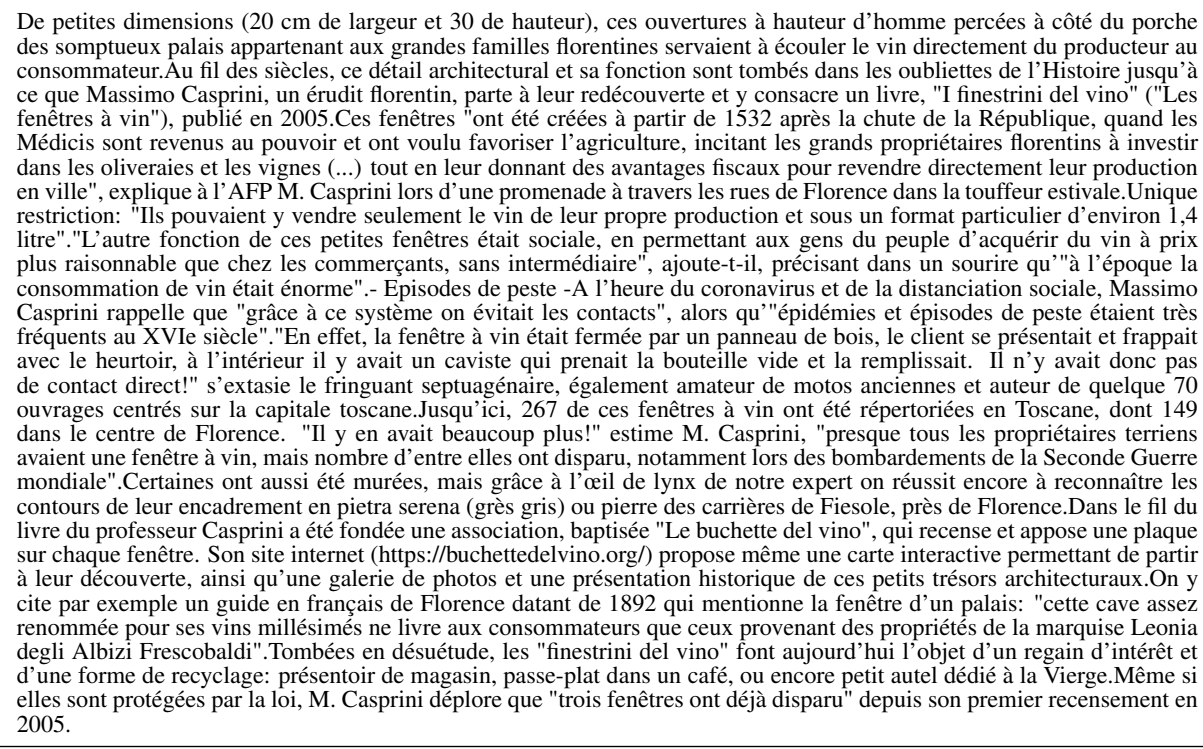 \\
\hline & mBARThez & $\begin{array}{l}\text { Florence, joyau de la Renaissance, peut s'enorgueillir d'un patrimoine mondi- } \\
\text { alement célèbre, mais dont certains détails restent encore aujourd'hui méconnus: } \\
\text { c'est le cas des discrètes "fenêtres à vin" ornant la façade de certains palais, qui } \\
\text { permettaient la vente de vin "sans contact", un concept redevenu d'actualité en } \\
\text { ces temps de coronavirus. } \\
\text { "A l'heure du coronavirus et de la distanciation sociale, il n'y avait pas de } \\
\text { contact direct !" A Florence, des fenêtres à vin, remplacées par des pierres, } \\
\text { auraient été oubliées dans lesoubliettes de l'Histoire. } \\
\text { Massimo Casprini, spécialiste des fenêtres à vins, est revenu mercredi sur la } \\
\text { révolution de Florence (Italie) où il a redécouvert l'existence de ces ouvertures } \\
\text { en plein air et à ciel ouvert qui permettaient, autrefois, à des propriétaires de } \\
\text { vins de revendre leur production à la ville. } \\
\text { De } 1532 \text { à nos jours, les fenêtres à vin des palais anciens de Florence sont les } \\
\text { plus souvent murées, un détail qui a sans doute survécu à l'épidémie de nouveau } \\
\text { coronavirus. } \\
\text { Au lieu de la pandémie de coronavirus, un jardin italien a retrouvé des crus du } \\
\text { monde entier: ils étaient des caves à vin français, à quelques dizaines de mètres } \\
\text { du sol, pour ne pas être contaminés par le Covid-19. }\end{array}$ \\
\hline & $\begin{array}{r}\text { Gold } \\
\text { mBART } \\
\text { mBARThez } \\
\text { BARThez } \\
\text { C2C }\end{array}$ & $\begin{array}{l}\text { Virus: comment la Florence des Médicis inventa la vente de vin "sans contact" } \\
\text { Les fenêtres à vin sont tombées dans les oubliettes de l'Histoire } \\
\text { Florence: les fenêtres à vin cachées dans un livre } \\
\text { Florence: des fenêtres à vin traditionnelles à l'heure des peste } \\
\text { Les fenêtres de la Florence en "huile de vin" : actualité automobile, infos, scoop }\end{array}$ \\
\hline
\end{tabular}

Table 14: C2C stands for CamemBERT2CamemBERT. OrangeSum document 34657. 


\begin{tabular}{|c|c|c|}
\hline & Document & 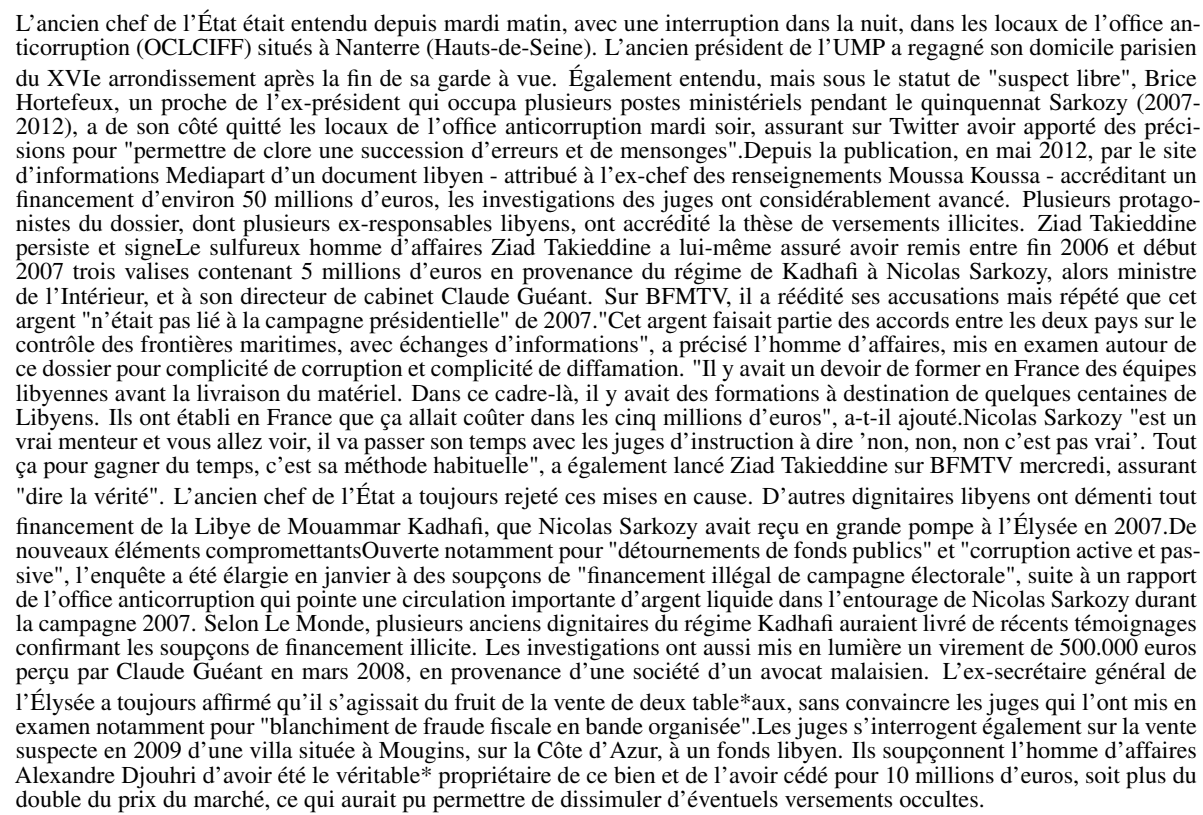 \\
\hline & mBARThez & $\begin{array}{l}\text { Après une vingtaine d'heures, la garde à vue de Nicolas Sarkozy s'est achevée } \\
\text { mercredi soir. L'ancien président a été mis en examen pour "corruption passive", } \\
\text { "financement illégal de campagne électorale" et "recel de fonds publics libyens" } \\
\text { et placé sous contrôle judiciaire dans le cadre de l'enquête sur des soupçons de } \\
\text { financement de sa campagne présidentielle de } 2007 \text { par la Libye de Mouammar } \\
\text { Kadhafi. } \\
\text { Ziad Takieddine, mis en examen autour de l'affaire des soupçons de financement } \\
\text { libyen de la campagne présidentielle de } 2007 \text { de Nicolas Sarkozy, a de nouveau } \\
\text { quitté les locaux de son établissement, à Nanterre, dans la nuit de mardi à } \\
\text { mercredi. } \\
\text { Cinq jours après la révélation d'un document par Mediapart, l'ancien président } \\
\text { de l'UMP et principal suspect dans l'affaire des soupçons de financement libyen } \\
\text { de sa campagne présidentielle de } 2007 \text { a quitté mardi soir les locaux où il était } \\
\text { auditionné. Brice Hortefeux et Claude Guéant ont apporté des précisions. } \\
\text { L'ancien président de la République Nicolas Sarkozy a quitté mardi matin } \\
\text { les locaux de l'office anticorruption où il était entendu. Les soupçons de } \\
\text { financement libyen de sa campagne présidentielle de } 2007 \text {. } \\
\text { Nicolas Sarkozy est mis en examen dans le cadre de l'enquête sur les soupçons } \\
\text { de financement libyen de sa campagne présidentielle de } 2007 \text {. Selon plusieurs } \\
\text { médias, l'ancien chargé de mission a dit mercredi n'être "pas au courant" de ce } \\
\text { que l'ex- }\end{array}$ \\
\hline & $\begin{array}{r}\text { Gold } \\
\text { mBART } \\
\text { mBARThez } \\
\text { BARThez } \\
\text { C2C }\end{array}$ & $\begin{array}{l}\text { Soupçons de financement libyen : Nicolas Sarkozy mis en examen } \\
\text { Affaire libyenne : Nicolas Sarkozy en garde à vue } \\
\text { Affaire libyenne : Nicolas Sarkozy entendu par les juges } \\
\text { Nicolas Sarkozy en garde à vue, la piste d'un financement libyen s'éloigne } \\
\text { Nicolas Sarkozy est "un vrai traître" selon l'entourage de Nicolas Sarkozy }\end{array}$ \\
\hline
\end{tabular}

Table 15: C2C stands for CamemBERT2CamemBERT. OrangeSum document 22208. 


\begin{tabular}{|c|c|c|}
\hline & Document & $\begin{array}{l}\text { Jean-Paul Dufrègne a passé un sale quart d'heure sur les réseaux sociaux } \\
\text { mercredi soir. Cet élu communiste de l'Allier a été filmé par les caméras } \\
\text { de TF1, dans un reportage diffusé le } 4 \text { avril au journal de } 20 \text { heures. Mais } \\
\text { téléspectateurs et internautes n'ont nullement prêté attention aux arguments } \\
\text { du député sur les inquiétudes persistantes des territoires ruraux et la réforme } \\
\text { institutionnelle sur laquelle planche le gouvernement. Non, ils étaient bien } \\
\text { trop captivés par son compteur de vitesse, filmé le temps de quelques plans } \\
\text { par les caméras de la première chaîne, comme le relève LCI.Car, sur une } \\
\text { route départementale limitée à } 90 \mathrm{~km} / \mathrm{heure,} \mathrm{Jean-Paul} \mathrm{Dufrègne} \mathrm{avait} \mathrm{le} \mathrm{pied} \\
\text { au plancher. Son compteur affichait } 124 \mathrm{~km} / \mathrm{heure,} \mathrm{plus} \mathrm{de} 30 \mathrm{~km} / \mathrm{heure} \text { au- } \\
\text { dessus de la limite autorisée. Une infraction que n'ont pas manqué de relever } \\
\text { de nombreux internautes. " Trois points et } 135 \text { euros d'amende ", note un } \\
\text { utilisateur de Twitter. " Bonjour, les limitations de vitesse ne s'appliquent pas } \\
\text { aux parlementaires? ", ironise un autre.Opposant au } 80 \mathrm{~km} / \text { heureCertains ont } \\
\text { par ailleurs fait le lien entre les positions politiques de l'élu communiste et cet } \\
\text { excès de vitesse. Car Jean-Paul Dufrègne est un farouche opposant au projet du } \\
\text { gouvernement de limiter le réseau français de routes secondaires à } 80 \mathrm{~km} / \mathrm{heure} \text {. } \\
\text { Avec une trentaine d'autres élus du Massif Central, il avait d'ailleurs adressé } \\
\text { une lettre ouverte à Emmanuel Macron sur le sujet, dénonçant une mesure " } \\
\text { injuste et pénalisante ", et un frein au développement du Massif Central. }\end{array}$ \\
\hline & $\begin{array}{r}\text { mBART } \\
\text { mBARThez } \\
\text { BARThez }\end{array}$ & $\begin{array}{l}\text { Jean-Paul Dufrègne, élu de l'Allier, a fait sensation sur les réseaux sociaux } \\
\text { après être apparu dans un reportage de TF1. On le voyait rouler à plus de } 120 \\
\mathrm{~km} / \text { heure sur une route secondaire. } \\
\text { L'élu communiste de l'Allier a été filmé le } 4 \text { avril au journal de } 20 \text { heures de } \\
\text { TF1, comme le relève LCI. Son compteur affichait } 124 \mathrm{~km} / \mathrm{heure} \text { au-dessus de } \\
\text { la limite autorisée. } \\
\text { Son compteur affichait } 124 \mathrm{~km} / \mathrm{h} \text { eure au-dessus de la limite autorisée. } \\
\text { Un élu communiste de l'Allier a été filmé le temps d'un reportage par les } \\
\text { caméras de TF1, dans la soirée du } 4 \text { avril, alors qu'il roulait } 90 \mathrm{~km} / \mathrm{heure} \\
\text { au-dessus de la limite autorisée. } \\
\text { Un député communiste du Puy-de-Dôme n'a pas manqué de se faire remarquer } \\
\text { en pleine séance de questions au gouvernement. Il était l'invité de Jean-Marc } \\
\text { Remontini, l'élu apparenté du PCF, qui a fait l'éloge de }\end{array}$ \\
\hline & $\begin{array}{r}\text { Gold } \\
\text { mBART } \\
\text { mBARThez }\end{array}$ & $\begin{array}{l}\text { Un élu épinglé à } 124 \mathrm{~km} / \mathrm{heure} \text { sur une route limitée à } 90 \\
\text { Un élu communiste flashé à } 124 \mathrm{~km} / \mathrm{heure} \text { sur TF1 } \\
\text { Un élu communiste flashé à } 124 \mathrm{~km} / \mathrm{heure} \text { sur TF1 } \\
\text { Un élu communiste passe un sale quart d'heure à cause de son compteur de } \\
\text { vitesse } \\
\text { Ce député du Doubs qui n'a plus le temps de répondre aux radars }\end{array}$ \\
\hline
\end{tabular}

Table 16: C2C stands for CamemBERT2CamemBERT. OrangeSum document 22077. 


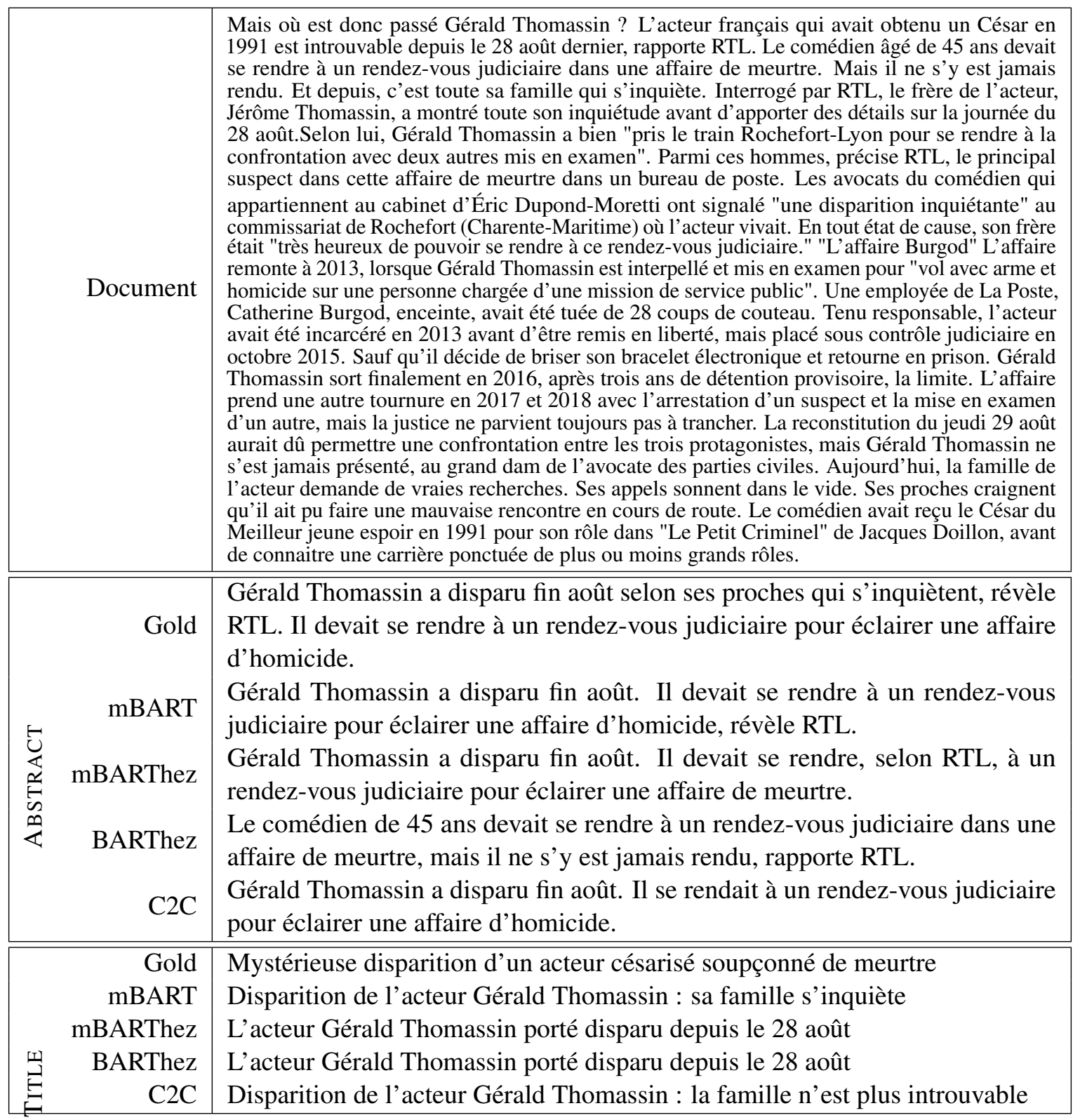

Table 17: C2C stands for CamemBERT2CamemBERT. OrangeSum document 22168. 


\begin{tabular}{|c|c|c|}
\hline & Document & 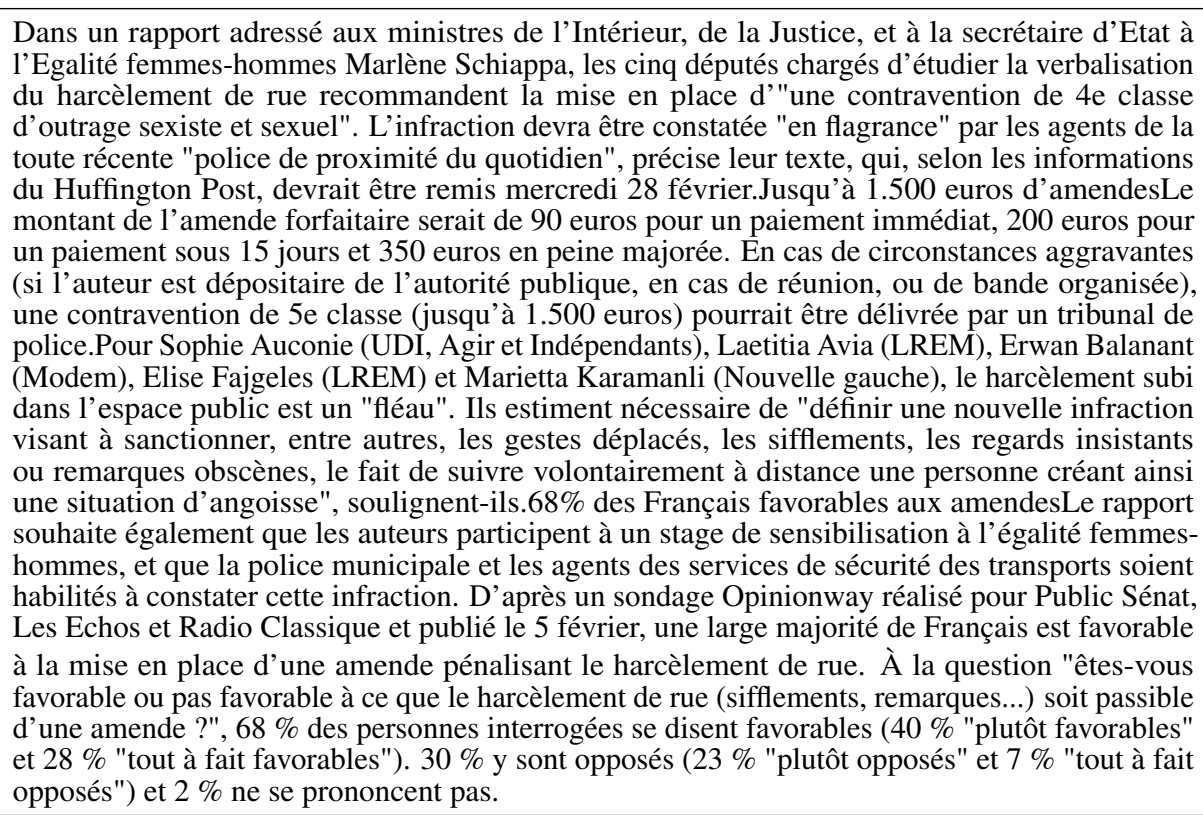 \\
\hline & mBARThez & $\begin{array}{l}\text { Des parlementaires préconisent de créer une infraction d'"outrage sexiste" } \\
\text { sanctionnant d'une amende immédiate de } 90 \text { euros "tout propos, comportement } \\
\text { ou pression à caractère sexiste ou sexuel" dans l'espace public. } \\
\text { Selon un rapport, dévoilé par le Huffington Post, le gouvernement envisage une } \\
\text { amende forfaitaire de } 90 \text { euros pour lutter contre le harcèlement de rue. En } \\
\text { cas de circonstances aggravantes, elle pourrait être délivrée par un tribunal de } \\
\text { police. } \\
\text { Dans un rapport adressé aux ministres de l'Intérieur, de la Justice et à la } \\
\text { secrétaire d'Etat à l'Egalité femmes-hommes, les députés chargés d'étudier } \\
\text { la verbalisation du harcèlement de rue recommandent la mise en place d'une } \\
\text { contravention de } 4 \text { e classe. } \\
\text { D'après un sondage Opinionway réalisé pour Public Sénat, Les Echos et Radio } \\
\text { Classique, une large majorité de Français sont favorables à la mise en place } \\
\text { d'une amende pénalisant le harcèlement de rue. } \\
\text { Selon un sondage Elabe pour Le Huffington Post, } 54 \% \text { des Français sont } \\
\text { opposés au projet de loi sur le harcèlement de rue. Une première en soi, alors } \\
\text { que la question de l'emprise sexuelle se pose déjà : les contraventions seront en } \\
\text { effet posées }\end{array}$ \\
\hline & $\begin{array}{r}\text { Gold } \\
\text { mBART } \\
\text { mBARThez } \\
\text { BARThez } \\
\text { C2C }\end{array}$ & $\begin{array}{l}\text { Harcèlement de rue : bientôt une amende immédiate de } 90 \text { euros ? } \\
\text { Harcèlement de rue : vers une contravention de } 4 \text { e classe ? } \\
\text { Harcèlement de rue : vers une contravention de } 4 \text { e classe ? } \\
\text { Harcèlement de rue : vers une contravention de } 4 \text { e classe ? } \\
\text { Harcèlement de rue : un rapport préconise une amende de } 5 \text { à } 5 \text { euros }\end{array}$ \\
\hline
\end{tabular}

Table 18: C2C stands for CamemBERT2CamemBERT. OrangeSum document 22423. 


\begin{tabular}{|c|c|c|}
\hline & Document & $\begin{array}{l}\text { Le } 18 \text { octobre dernier, Jacline Mouraud se faisait connaître en publiant sur } \\
\text { Facebook une vidéo dans laquelle elle poussait un "coup de gueule" contre } \\
\text { le gouvernement. Aujourd'hui, la Bretonne a pris ses distances par rapport } \\
\text { au mouvement, notamment face à d'autres figures plus radicales comme Éric } \\
\text { Drouet.Jacline Mouraud réfléchit désormais à créer son propre parti, "la seule } \\
\text { chose envisageable", comme elle l'explique au JDD.Nicolas Sarkozy, "le seul } \\
\text { qui a des couilles"Cette figure des "gilets jaunes", accusée de faire le jeu de } \\
\text { LREM estime que "le problème" d'Emmanuel Macron "c'est qu'il est jeune". } \\
\text { "Il devrait y avoir un âge minimum pour être président : } 50 \text { ans", souligne } \\
\text { Jacline Mouraud.Dans le JDD, elle raconte d'ailleurs avoir voté blanc lors de la } \\
\text { dernière présidentielle. En } 2007 \text { et 2012, c'est Nicolas Sarkozy, "le seul qui a } \\
\text { des couilles", que la figure des "gilets jaunes" avait soutenu. En attendant de se } \\
\text { lancer, pas question pour elle en tous les cas d'être candidate aux européennes } \\
\text { sur une liste de La République en marche. }\end{array}$ \\
\hline & Gold & L'une des figures du mouvement ne sera toutefois pas candidate aux prochaines \\
\hline & mBART & $\begin{array}{l}\text { Jacline Mouraud, figure des "gilets jaunes", estime que le président d'Emmanuel } \\
\text { Macron est trop jeune pour être président. }\end{array}$ \\
\hline & mBARThez & $\begin{array}{l}\text { Dans un entretien au JDD, la figure des "gilets jaunes" Jacline Mouraud révèle } \\
\text { qu'elle réfléchit à créer son propre parti. }\end{array}$ \\
\hline $\begin{array}{l}n \\
\infty \\
\infty \\
\&\end{array}$ & BARThez & $\begin{array}{l}\text { Dans les colonnes du JDD, la figure des "gilets jaunes" explique qu'elle envisage } \\
\text { de se présenter aux européennes sur une liste La République en marche. }\end{array}$ \\
\hline & $\mathrm{C} 2 \mathrm{C}$ & $\begin{array}{l}\text { Retirée de la vie politique depuis plusieurs mois, Bretone Mouraud envisage } \\
\text { de se lancer en politique. Et elle réfléchit à quelque chose de plus, rapporte le } \\
\text { JDD. }\end{array}$ \\
\hline & Gold & "Gilets jaunes" : Jacline Mouraud réfléchit à créer son parti \\
\hline & mBART & "Gilets jaunes" : Jacline Mouraud lance son propre parti \\
\hline & mBARThez & "Gilets jaunes" : Jacline Mouraud prend ses distances \\
\hline & BARThez & La figure des "gilets jaunes" Jacline Mouraud va créer son propre parti \\
\hline$E$ & $\mathrm{C} 2 \mathrm{C}$ & "Gilets jaunes" : Jacline Mouraud réfléchit à sa propre candidature \\
\hline
\end{tabular}

Table 19: C2C stands for CamemBERT2CamemBERT. OrangeSum document 19233. 


\begin{tabular}{|c|c|c|}
\hline & Document & 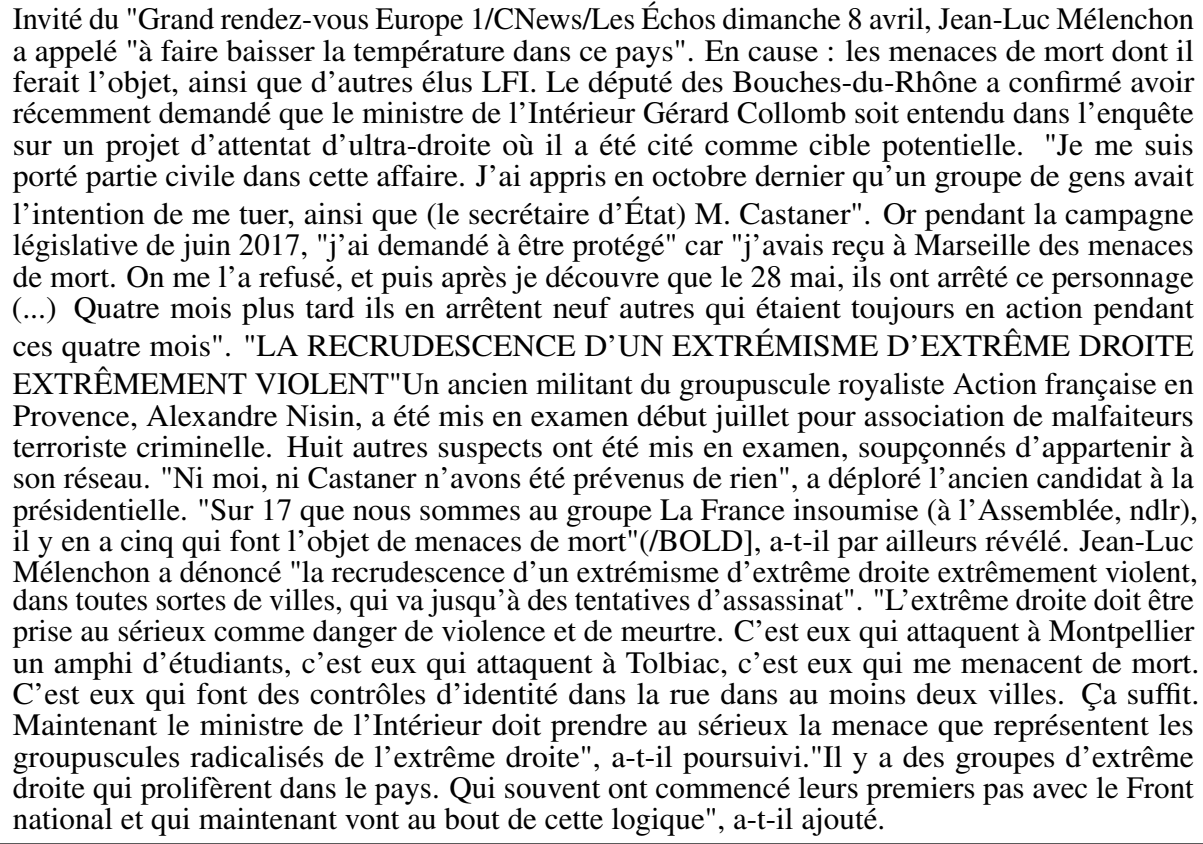 \\
\hline & mBARThez & $\begin{array}{l}\text { Le leader de La France insoumise (LFI) dénonce la "recrudescence" d'une } \\
\text { "extrême droite extrêmement violent(e)" en France, qui doit être "prise au } \\
\text { sérieux" par le gouvernement. } \\
\text { S'il dénonce la recrudescence d'un extrémisme d'extrême droite "extrêmement } \\
\text { violent" dans certaines villes, le chef de file de La France insoumise (LFI), } \\
\text { Jean-Luc Mélenchon, s'est attaqué au ministre de l'Intérieur, Gérard Collomb. } \\
\text { Au micro d'Europe } 1 \text { dimanche } 8 \text { avril, Jean-Luc Mélenchon a réagi aux men- } \\
\text { aces de mort dont il fait l'objet et dénoncé "la recrudescence d'un extrémisme } \\
\text { d'extrême droite extrêmement violent". } \\
\text { - Le chef de file de La France insoumise et ancien candidat à la présidentielle } \\
\text { est vent debout contre le projet d'attentat déjoué à Marseille. Il estime que } \\
\text { le ministre de l'Intérieur, Gérard Collomb, est menacé de mort par un groupe } \\
\text { d'extrême droite. } \\
\text { Selon le leader de La France insoumise (LFI), le député des Bouches-du- } \\
\text { Rhône, Jean-Luc Mélenchon, "rappelle à tous ceux suspectés d'avoir menacé } \\
\text { d'assassiner le ministre de l'Intérieur, ce que conteste le parti et }\end{array}$ \\
\hline & $\begin{array}{r}\text { Gold } \\
\text { mBART } \\
\text { mBARThez } \\
\text { BARThez }\end{array}$ & $\begin{array}{l}\text { VIDÉO. Cinq députés de La France insoumise font l'objet de menaces de mort, } \\
\text { selon Jean-Luc Mélenchon } \\
\text { Menaces de mort : Jean-Luc Mélenchon s'en prend à Castaner } \\
\text { Jean-Luc Mélenchon dénonce les "menaces de mort" de Gérard Collomb } \\
\text { VIDÉO. Jean-Luc Mélenchon dénonce les menaces de mort dont Gérard Col- } \\
\text { lomb est victime } \\
\text { Projet VIDÉO. Menace de mort à Marseille : Jean-Luc Mélenchon menace de } \\
\text { démissionner }\end{array}$ \\
\hline
\end{tabular}

Table 20: C2C stands for CamemBERT2CamemBERT. OrangeSum document 22060. 